\title{
Die historischen Hintergründe des irakischen Anspruchs auf Kuwait
}

Von René Klaff

I.

In den frühen Morgenstunden des 2. August 1990 überfielen irakische Truppen Kuwait, vertrieben die dortige Regierung und besetzten den kleineren Nachbarstaat mit dem Ziel, ihn vollständig in den Irak zu integrieren. Diese für die Weltöffentlichkeit völlig überraschende Aktion löste eine der schwersten internationalen Krisen seit dem Ende des Zweiten Weltkrieges aus; der massive Protest der Weltstaatengemeinschaft gegen die Annexion Kuwaits kam in zwölf Resolutionen des UN-Sicherheitsrates zum Ausdruck, in denen der Rückzug der irakischen Truppen und die Wiederherstellung der kuwaitischen Souveränität gefordert wurde. Die Weigerung des irakischen Präsidenten, Saddam Hussein, diese Beschlüsse zu befolgen, führte schließlich im Januar 1991 zu einer großangelegten militärischen Aktion gegen den Irak. Einer internationalen Streitmacht unter der Führung der USA gelang es dabei auf gewaltsamem Wege, den Irak zur Annahme der Resolutionen und zur Räumung Kuwaits zu zwingen, womit - zumindest unter völkerrechtlichen Gesichtspunkten - der status quo ante wiederhergestellt wurde.1

Die Motive, die Saddam Hussein zur Einverleibung Kuwaits trieben, sind vielfältig und im einzelnen in diesem Beitrag nicht näher darzustellen. Sie liegen vor allem in der strategischen Bedeutung, die der kleine, ölreiche Nachbarstaat für den Irak hat. Mit dem Besitz von Kuwait erhielte der Irak den langersehnten Zugang zum Persisch/Arabischen Golf, der ihm aufgrund seiner nur ca. 50 Meilen langen, für den Bau von Tiefwasserhäfen ungeeigneten Küstenlinie bislang faktisch fehlte. Darüber hinaus schien der Zugriff auf die

1 Die Kampfhandlungen zur Durchsetzung der UN-Sicherheitsratsresolutionen begannen in der Nacht vom 16./17.1.1991 und wurden am 28.2.1991 nach ihrer bedingungslosen Annahme durch den Irak eingestellt. Zu den Resolutionen vgl. United Nations Department of Public Information: United Nations Security Council Resolutions Relating to the Crisis in the Persian Gulf, DPI/1 104 Corr. 1/December 1990. Für eine ausführliche Darstellung der Ereignisse zwischen August 1990 und Januar 1991 vgl. Gunther Hellmann: Der Krieg um Kuwait: Katalysator einer "neuen Weltordnung" oder Vorbote neuer Konflikte?, in: Aus Politik und Zeitgeschichte (APuZ), Bd. 7-8, 1991, S. 12-26. 
Reichtümer Kuwaits die großen finanziellen Probleme lösen zu können, die durch die hohe Verschuldung Iraks infolge des achtjährigen Krieges mit dem Iran entstanden waren.2

Nach den Regeln des Völkerrechts läßt sich die gewaltsame Annexion Kuwaits in keinem Falle legitimieren: Kuwait hat als souveräner Staat Anspruch auf territoriale Unversehrtheit, die ihm z.B. durch die Charta der Vereinten Nationen und die der Arabischen Liga zugesichert ist. Irak, Mitglied in diesen beiden Staatengemeinschaften, hat mit seiner militärischen Aktion offensichtlich Rechtsbruch begangen.

Dennoch hat Saddam Hussein versucht, eine Legitimationsgrundlage für seinen Schritt zu konstruieren. Die Begründungen, die er für die Besetzung und anschließende Annexion des kleinen Nachbarstaates als 19. Provinz des Iraks3 vorgebracht hat, betreffen die historischen Beziehungen beider Länder. Seine Argumentation läßt sich in zwei wesentliche Punkte zusammenfassen:

1. Kuwait sei in der Vergangenheit Bestandteil der osmanischen Provinz von Basra gewesen; rechtmäßigerweise müsse Kuwait daher heute zum Irak gehören, dem Nachfolgestaat des Osmanischen Reiches in dem betreffenden Gebiet.

2. Kuwait sei ein künstlicher Staat, seine Selbständigkeit nur die Folge illegitimen britischen Kolonialverhaltens. 4

In der arabischen Welt - wenn nicht bei den einzelnen Regimen, so doch beim Großteil der arabischen Massen - wird diese Darstellung weitgehend akzeptiert.5 Viele Araber sehen in

2 Zu den politischen Auslösefaktoren vgl. Bassam Tibi: Die irakische Kuwait-Invasion und die Golfkrise, in: Beiträge zur Konfliktforschung, Nr. 4, 1990, S. 5-34, hier S. 11 ff., und ders.: Der Irak und der Golfkrieg. Regionale Faktoren der irakischen Invasion Kuwaits, in: APuZ, Bd. 7-8, 1991, S. 3-11; Ursula Braun: Epizentrum Kuwait: Die weltpolitische Dimension eines Regionalkonfliks, in: Aussenpolitik, Jg. 42, 1991, Heft 2, S. 59-68, hier S. 59 ff. Schätzungen zur Schuldenlast des Iraks liegen zwischen 70-120 Mrd. \$; die Höhe der an Kuwait zurückzuzahlenden Kredite belaufen sich allein auf ca. $30 \mathrm{Mrd}$.\$. Vgl. Liesl Graz: Iraqi Sabres Rattle in the Gulf, in: Middle East Intemational (MEI), No. 381 (3.8.1990), S. 3 ff.; Anthony McDermott: Iraq and Kuwait - Two Vulnerable Economies, in: MEI, No. 383 (14.9.1990), S. 19.

3 Zur 19. Provinz des Irak wurde Kuwait of fiziell am 28.8.1990 erklärt, allerdings nur der südliche Teil des Landes um die Hauptstadt Kuwait City. Die nördliche Hälfte wurde in die Verwaltungsstruktur der irakischen Provinz von Basra integriert. Vgl. NZZ, 4.8.1990 (A. Hottinger: "Kuwait als Provinz in den Irak eingegliedert"); Text des irakischen Dekrets in al-Muntada (Amman), September 1990, zusammengef a Bt bei Tibi, Golfkrise, S. 9.

4 Vgl. z.B. Tibi, ebda. S. 9 u. Fn 26, S. 30; NZZ, 12./13.8.1990 (A. Hottinger: "Gipfeltreffen der letzten Hoffnung in Kairo").

5 Vgl. NZZ, 15.8.1990 ("Bewunderung der Palästinenser für Saddam"); NZZ, 16.8.1990 ("Spaltung und Venwirnung in der arabischen Welt"); NZZ, 26./27.8.1990 ("Rückhalt Saddam Husseins im 
der Invasion und Annexion Kuwaits durch Irak daher eine legitime Aktion. Schon aus diesem Grunde bedarf der Versuch Saddam Husseins, seinen Schritt mit historischen und völkerrechtlichen Argumenten zu rechtfertigen, einer näheren Untersuchung. Um die Legitimität der irakischen Ansprüche auf Kuwait beurteilen zu können, müssen folgende Fragen beantwortet werden:

1. War Kuwait integraler Bestandteil des Osmanischen Reiches, konkret der Provinz von Basra?

2. Kann der Irak daher als Nachfolgestaat des Osmanischen Reiches Ansprüche auf Kuwait legitimerweise vorbringen?

3. Hat der Irak diese Ansprüche kontinuierlich vertreten?6

Ш.

Diese Fragen führen in die Anfänge der Geschichte Kuwaits zurück. Im Jahre 1756 ließen sich mehrere Beduinenstämme aus dem zentralen Hochland der arabischen Halbinsel an dem Küstensaum nieder, der heute Bucht von Kuwait genannt wird. Ihr Herkunftsgebiet im Innern Arabiens hatten sie wegen anhaltender Dürre bereits Jahre vorher verlassen müssen. Ihre neue Heimat bef and sich an der Stelle eines verlassenen portugiesischen Handelsstützpunktes, der der neuen Siedlung indirekt ihren Namen gab: Kuwait bedeutet auf arabisch "kleines Kastell / kleines Fort". Zum politischen Oberhaupt wählten die Stämme den Scheich des Clans der Al Sabah; seither herrscht diese Dynastie in Kuwait. Im Jahre 1961, dem Zeitpunkt der völligen Unabhängigkeit, nahmen die Sabah-Scheichs den Titel "Emir" an. 7

Das Siedlungsgebiet an der Bucht von Kuwait gehörte nominell zum Osmanischen Reich. Bereits seit der Mitte des 16. Jahrhunderts beanspruchten die Osmanen, damals unter dem Sultan Sulaiman dem Prächtigen (1520-1566), die Küsten der Arabischen Halbinsel und weite Teile ihrer bewohnbaren Zentrallandschaften als zum Reich von Konstantinopel

Maghreb"); Peter Mansfield: The Arab Nation and Saddam Hussein, in: MEI, No. 382 (31.8.1990), S. 26 f.; Ghada Karmi: Arab Reactions to the Gulf Crisis, in: MEI, No. 384 (28.9.1990), S. 20.

6 Vgl. Ulrich Gehrke / Gustav Kuhn: Die Grenzen des Irak. Historische und rechtliche Aspekte des irakischen Anspruches auf Kuwait und des irakisch-persischen Streits um den Schatt al-Arab, Stuttgart 1963, S. 31-40 u. S. 119 f.; Husain M. Albaharna: The Arabian Gulf States. Their Legal and Political Status and their Intemational Problems, 2. rev. ed., Beirut 1975, S. 252; R. V. Pillai / Mahendra Kumar: The Political and Legal Status of Kuwait, in: Intemational and Comparative Law Quarterly, Bd. 11, 1962, S. 108-130, hier S. 116.

7 Zur Geschichte Kuwaits vgl. z.B. H. R. P. Dickson: Kuwait and her Neighbours, London 1956; Encyclopédie de l'Islam, Nouvelle Edition (EI 2), Bd. 5, Leiden/Paris 1988, Art. Kuwayt (R. M. Burell), S. 576 ff. Zur Entwicklung der modemen staatlichen Gebilde am Golf vgl. Rosemarie Said Zahlan: The Making of the Modem Gulf States, London 1989, S. $12 \mathrm{ff}$. u. S. $25 \mathrm{ff}$. zum Verhältnis der einzelnen kuwaitischen Clans untereinander. 
gehörend. ${ }^{8}$ Die Kuwaitis akzeptierten diesen Anspruch. Scheich Sabah reiste noch im Jahre 1756 zum osmanischen Provinzgouverneur nach Basra, um die friedlichen Absichten der neuen Bewohner darzulegen, gleichzeitig aber auch, um die Oberhoheit des osmanischen Sultans zu garantieren. Ein konkreter Abhängigkeitsstatus von Kuwait zum Osmanischen Reich wurde bei dieser Gelegenheit nicht festgelegt.9 Damit begann die erste Phase der kuwaitischen Geschichte, die bis zum formalen Beginn des Engagements des Britischen Empires 1899 andauerte.

Bereits in dieser Zeit bildeten sich die Grundstrukturen kuwaitischer Autonomie heraus. Zwar erkannten die Al Sabah bei verschiedenen Gelegenheiten die Oberhoheit des Osmanischen Reiches an. Dies allein läßt jedoch über das faktische Verhältnis zwischen dem Reich - bzw. den Gouverneuren von Baghdad und Basra als dessen Vertreter in der Region - und Kuwait noch keine Aussage zu. Die nominelle Anerkennung der Oberhoheit des Osmanischen Reiches hatte nämlich keine politischen Konsequenzen. Die Türken waren an dieser Peripherie ihres Reiches nicht in der Lage, politische Hoheitsgewalt auszuüben. Folglich besaß Kuwait, d.h. der regierende Scheich der Al Sabah Dynastie, völlige Autonomie. Das Verhältmis, in dem Kuwait tatsächlich zum Osmanischen Reich stand, blieb daher ungeklärt. Daß Kuwait integraler Bestandteil des Osmanischen Reiches sei, ergab sich allein aus dessen Anspruch; eingelöst, etwa in Form einer militärischen Präsenz oder einer Ausdehnung der osmanischen Verwaltung, wurde dieser Anspruch nie, weder in der Zeit vor den Al Sabah noch seit 1756.10

Erst im letzten Drittel des 19. Jahrhunderts wurden emstzunehmende Versuche unternommen, eine festere Bindung Kuwaits an das Reich herzustellen. Im Jahre 1871 reist mit Midhat Pascha zum ersten Mal ein osmanischer Gouverneur nach Kuwait. Es gelang ihm, mit Scheich Abdallah ein Abkommen auszuhandeln. Danach wurde dem Scheich der Titel eines Distriktstatthalters - eines Qaimmaqams - verliehen; das Osmanische Reich betrachtete den Scheich somit als obersten Beamten eines Qazas, einer türkischen Verwaltungseinheit.11 Gleichzeitig übernahm der Scheich die Flagge des Osmanischen Reiches und

8 Die osmanischen Truppen eroberten 1526 die Küsten des Jemen, 1534 Baghdad und Basra, 1538 Aden; aus diesen Eroberungen leiteten sie die beschriebenen Ansprüche ab. Vgl. dazu vor dem Hintergrund der Geschichte Iraks: Stephen H. Longrigg: Four Centuries of Modem Iraq, Oxford 1925, S. 1 ff. Allerdings war die Herrschaft der Osmanen gerade im Südirak nicht kontinuierlich gesichert, vgl. ebda., S. 123 ff. Im 16. Jh. entbrannte auch die Rivalität europäischer Kolonialmächte (Portugal, Holland, Großbritannien) um Handelsstützpunkte ind Einflußzonen in der GolfRegion.

9 Vgl. Dickson, Kuwait, S. 26 f.

$10 \mathrm{Vgl}$. Gehrke/Kuhn, Irak, S. $40 \mathrm{ff}$. und die Beurteilung des Verhältnisses Kuwaits zum Osmanischen Reich ebda., S. 121 ff. Auch Pillai/Kumar, Status of Kuwait, S. 109 f.

11 Die größte territoriale Verwaltungseinheit im Osmanischen Reich war die Provinz (Wilayet), die wiederum aus mehreren Sanjaks bestand; oberste Beamte waren der Wali bzw. der Mutassarif. Die nächst kleineren Einheiten - ursprünglich Gerichtsbezirke - waren der Qaza (unter der Leitung 
erkannte die Oberhoheit des Sultans in Konstantinopel an. Als Gegenleistung erhielt er die Zusicherung der völligen inneren Autonomie und der Erbfolge der Al Sabah Dynastie in ihrem Scheichtum. Nach osmanischer Interpretation wurde mit diesem Abkommen der Status Kuwaits als Qaza der Provinz von Basra formalisiert.12

De facto bedeutete dieses Abkommen jedoch die Festschreibung der Situation, die seit 1756 bestanden hatte. Die Al Sabah blieben selbstverantwortlich, ihre politische Macht und ihre Ordnungsfunktionen nahmen sie weiterhin aus eigener Kraft wahr. An diesem Sachverhalt änderte sich auch durch das Abkommen mit Midhat Pascha trotz gegenläufiger Tendenzen zwischen 1871 und 1899 grundsätzlich nichts. 13

Das Verhältnis Kuwaits zum Osmanischen Reich ist in dieser ersten Phase seiner Geschichte daher folgendermaßen zu beurteilen:

1. Das Osmanische Reich hat weder vor der Etablierung der Al Sabah Dynastie noch danach je tatsächlich politische Macht in Kuwait ausgeübt. Über die Formulierung eines Anspruches hinaus konnte das Osmanische Reich keine praktischen Schritte zur Integration Kuwaits in seinen unmittelbaren Herrschaftsbereich unternehmen.

2. Die Al Sabah übten seit 1756 selbständig Ordnungsfunktionen in Kuwait aus. Diese sind ihnen weder vom Osmanischen Reich übertragen worden, noch übten sie sie in dessen Namen aus. Daraus ergibt sich, daß Kuwait kein Verwaltungsdistrikt der osmanischen Provinz von Basra war. Sein Scheich war zu keiner Zeit Befehlsempfänger der Walis von Basra oder von Baghdad. Auch durch das Abkommen von 1871 wurde kein Abhängigkeitsverhältnis zu diesen Provinzen begründet.

3. Durch die Anerkennung der osmanischen Oberhoheit gehörte Kuwait zweifelsohne zum osmanischen Reichsverband. Allerdings war es nicht in die Hoheits- und Verwaltungsstrukturen des Reiches integriert. Im Gegenteil: Im Abkommen von 1871 wird Kuwait explizit innere Autonomie zugestanden. Daran ändert auch die Ưbertragung des

eines Qaimmaqam) und der Nahiye (i.d.R. unter der Leitung eines Mudir). Vgl. vor dem Hintergrund der Verwaltungsreformen des 19. Jh. H. A. R. Gibb / Harold Bowen: Islamic Society and the West, vol. 1, Part 1, London 1950, S. 138 ff.; EI 2, Bd. 4, Art. Kaim-makam (E. Kuran), S. 482; Enzyklopädie des Islam, (EI 2), Bd. 4, Leiden/Leipzig 1934, Art. Sandjak (J. Deuy), S. 159 ff. und Art. Wilaya (Heffening), S. $1231 \mathrm{f}$.

12 Vgl. den Uberblick über die Verwaltungsstruktur der Provinz von Basra bei Longrigg, Centuries, S. 313, Fn. 1; nach anderen Quellen war Kuwait mit diesem Schritt Sanjak der Provinz von Baghdad geworden, vgl. Gehrke/Kuhn, Irak, S. 129, Fn. 40.

13 Unter diesen "gegenläufigen Tendenzen" ist insbesondere die Politik von Schei Muhammad b. Sabah (1892-96) zu verstehen, der engeren Anschluß an das Osmanische Reich suchte. Zu den Hintergründen, die im Zusammenhang mit dem Besitz ertragreicher Dattelhaine in der Provinz von Basra stehen, vgl. Gehrke/Kuhn, Irak, S. 46 f.; Zahlan, Modem Gulf States, S. 26. 
Titels Qaimmaqam nichts. Politische Entscheidungen in Kuwait wurden auch weiterhin nicht im türkischen Namen getroffen; weder auf die Bestellung noch auf die Amtsführung des regierenden Scheichs gab es irgendeinen türkischen Einfluß. Durch die Verleihung dieses Titels wurde sein Träger somit nicht automatisch zur osmanischen Amtsperson. 14 Im übrigen weigerte sich Scheich Mubarak (1896-1915), diesen Titel zu tragen. 15

4. Vor diesem Hintergrund muß Kuwait als Vasall des Osmanischen Reiches bezeichnet werden16, nicht jedoch als dessen integraler Bestandteil. Das Osmanische Reich war Oberherr (Suzerän) des ansonsten autonomen Scheichturns von Kuwait; das Verhältnis des Suzeräns zum Vasallen ist nicht gleichbedeutend mit der Ausübung staatsrechtlicher Souveränität des ersteren über den zweiten, sondern schließt sie in der Regel - wie im Falle der Beziehung des Osmanischen Reiches zu Kuwait - aus. 17

\section{III.}

Am 23.1.1899 unterzeichnete Scheich Mubarak einen Geheimvertrag mit Großbritannien, der für die weitere Geschichte Kuwaits, aber auch für die heutige Argumentation Saddam Husseins von großer Bedeutung ist. In dem Vertrag verpflichtete Mubarak sich und seine Nachfolger, "... not to receive the agent or representative of any Power or Govemment at Kowait, or at any other place within the limits of his territory, without the previous sanction of the British govemment; and he further binds himself, his heirs and successors, not to cede, sell, mortgage, or give for occupation or for any other purpose, any portion of his territory to the Government or subjects of any other Power without the previous consent of Her Majesty's Government ...".18 Vom Britischen Empire erhielt Mubarak im Gegenzug neben einer Summe von 1000 Pfund die Zusicherung britischen Schutzes und "guter Dienste" für sich und seine Nachfolger.19

14 Vgl. Pillai/Kumar, Status of Kuwait, S. 117, auch für ähnlich gelagerte Fälle.

15 Vgl. Dickson, Kuwait, S. 136 f. Die Kuwaitis interpretierten den Titel Qaimmaqam bisweilen fälschlicherweise - als reinen Ehrentitel, ähnlich wie Pascha, Bey oder Effendi; vgl. Gehrke/Kuhn, Irak, S. 129.

16 So übereinstimmend Gehrke/Kuhn, Irak, S. 121 ff.; Albaharna, Gulf States, S. 253; Pillai/Kumar, Status of Kuwait, S. $116 \mathrm{ff}$.

17 Der Status der Vasallität bezieht sich auf Verpflichtungen wie Tributzahlungen, Heeresfolge und militärische Schutzfunktionen für die Sicherung des Reichsverbandes; vgl. dazu Gehrke/Kuhn, Irak, S. 136.

18 Vertrag zwischen Großbritannien und dem Scheich von Kuwait, in: Gehrke/Kuhn: Die Grenzen des Irak. Dokumentenanhang, Dokument 7, S. $12 \mathrm{f}$.

19 Zur britischen Diskussion über die Annäherung an Kuwait, die besonders vom Vizekönig in Indien, Lord Curzon, vorangetrieben wurde, vgl. Briton Cooper Busch: Britain and the Status of Kuwayt, 1896 - 1899, in: Middle East Joumal, vol. 21, 1967, S. 187-198, bes. S. 196 f. 
Mit diesem Vertrag wurde Kuwait faktisch ein Quasi-Protektorat Großbritanniens; ein formaler Protektoratsstatus wurde jedoch nicht formuliert.20 Die kuwaitischen Scheichs hatten während des ganzen 19. Jahrhunderts hindurch versucht, britischen Schutz zu erlangen, der sie insbesondere vor Eroberungszügen aus der arabischen Halbinsel bewahren sollte, denen Kuwait während des 19. Jahrhunderts permanent ausgesetzt war und die das Osmanische Reich nicht verhindern konnte.21 Scheich Mubarak wollte aber auch einer schleichenden Integration in die osmanische Provinz von Basra zuvorkommen, die Teile des Al Sabah Clans in Zusammenarbeit mit türkischen Kräften in Basra betrieben.22 Zur Konsolidierung seiner Herrschaft, die von Teilen seiner eigenen Familie und den Gegnern aus der arabischen Halbinsel bedroht war, hatte Mubarak sogar versucht, Kuwait unter ein formales britisches Protektorat zu bringen; diese Strategie behielt er in den folgenden Jahren, wenn auch erfolglos, bei. 23

Großbritannien seinerseits hatte bis zur Unterzeichnung des Vertrages vor direktem Engagement in Kuwait zurückgeschreckt. Erst der gegen Ende des 19. Jahrhunderts zunehmende Drang des russischen Reiches nach Süden, in die Richtung der warmen Gewässer des Indischen Ozeans und des Persischen Golfes, bewirkte einen Umschwung in der britischen Haltung. 24 Ein zweiter Grund waren die diversen Pläne zum Bau von Eisenbahnlinien innerhalb des Osmanischen Reiches. Neben französischen und russischen Vorhaben bezogen sich diese vor allem auf das von Deutschen vorangetriebene Projekt der sogenannten Baghdad-Bahn, die Berlin mit dem Osmanischen Reich verbinden sollte und als dessen Endbahnhof Kuwait im Gespräch war. 25 Somit rückte der Persische Golf und insbesondere Kuwait in die britische Interessensphäre. Der Vertrag mit Mubarak sollte Entwicklungen

$20 \mathrm{Zu}$ diesen in der Kolonialzeit nicht unüblichen Rechtsverhältnissen zwischen Schutzmacht und Quasi-Protektorat vgl. ausführlicher: Encyclopedia of Public International Law, publ. by Max Planck Institute for Comparative and Intemational Law, Amsterdam/New York/Oxford, Bd. 10, 1987, S. 336 ff., Art. Protectorate; Georg Dahm / Jost Delbrück / Rüdiger Wolfrum: Völkerrecht, Bd. I/1: Die Grundlagen. Die Völkerrechtssubjekte, 2. Aufl., Berlin/New York 1989, S. 226 f. Vgl. auch Pillai/Kumar, Status of Kuwait, S. $118 \mathrm{f}$., die die völkerrechtliche Legalität dieses Vertrages beanstanden.

21 Zur Auseinandersetzung mit den Wahhabiten bzw. den Al-Sa'ud im 19. und frühen 20. Jh. vgl. Dickson, Kuwait, Kap. 4, 5, 6. Seit Mitte der neunziger Jahre des 19. Jh. stellte für ungefähr ein Jahrzehnt das Schammar-Reich der Raschiden die größte Gefahr für Kuwait dar. Vgl. EI 1, Bd. 4, Art. Shammar (A. Guillaume), S. 330 f. In dieser Periode war Mubarak Al-Sabah mit den Al-Sa'ud praktisch verbündet. Vgl. Dickson, Kuwait, S. $136 \mathrm{ff}$.

22 Vgl. Dickson, Kuwait, S. $136 \mathrm{f}$.

23 Vgl. Gehrke/Kuhn. Irak, S. 67 ff.; zu Befürwortem und Gegnem eines solchen Schrittes unter den Briten vgl. hier und Busch, Status of Kuwayt, passim.

24 Vgl. Günther Stökl: Russische Geschichte. Von den Anfängen bis zur Gegenwart, 2. erw. Aufl., Stuttgart 1965, S. 517 ff. u. S. 527 ff.

25 Zur Baghdad-Bahn neuerdings sehr ausführlich Jürgen Lodemann / Manfred Pohl: Die Baghdadbahn. Geschichte und Gegenwart einer berühmten Eisenbahnlinie, Mainz 1989. 
vorbeugen, die einer anderen Macht als dem Britischen Empire Einfluß über das Scheichtum verschafft hätten. 26

Ein Nebeneffekt des Vertrages mit Großbritannien war die Begründung einer kuwaitischen Staatlichkeit. Scheich Mubarak handelte explizit für das von ihm beherrschte Territorium; damit übte er eine gebietsbezogene Hoheitsgewalt aus, im Unterschied zur bisherigen, auf den Stamm bezogenen, personalen Hoheitsgewalt der Al Sabah.27 Der Vertragspartner Großbritannien akzeptierte Mubarak als denjenigen, der aus originärer Macht heraus für die Geschicke Kuwaits verantwortlich war. Die türkischen Versuche, Kuwait in die Verwaltungsstruktur des Osmanischen Reiches wirksam zu integrieren, waren damit gescheitert; dies gilt auch für die später, nach Bekanntwerden des Geheimvertrages untemommenen Maßnahmen des Reiches. 28

Das formale Abhängigkeitsverhältnis des Staates Kuwait gegenüber dem Osmanischen Reich und dem Britischen Empire blieb indes undeutlich. Kuwait schied durch den Vertrag von 1899 nicht aus dem osmanischen Reichsverbund aus. Sowohl Großbritannien als auch Mubarak - der z.B. an der türkischen Flagge festhielt - bestätigten den Sultan von Konstantinopel weiterhin als Oberherrn Kuwaits. Kuwait war seit 1899 Vasallenstaat des Osmanischen Reiches unter besonderem britischen Schutz, dessen interne, innerstaatliche Autonomie dadurch jedoch nicht grundsätzlich berührt wurde.29 Vasallenstaaten hat das Osmanische Reich in allen Phasen seiner Geschichte gekannt, so daß der Status von Kuwait durchaus nicht als ungewöhnlich zu bezeichnen ist. 30

Dieser Status Kuwaits - als Vasall des Osmanischen Reiches und Quasi-Protektorat Großbritanniens - wurde in der "Anglo-Ottoman Draft Convention on the Persian Gulf Area" vom 29.7.1913 im Kem bestätigt. In dieser Konvention - die bis zum Ausbruch des 1. Weltkrieges im übrigen nicht ratifiziert wurde und danach gegenstandslos war - wurde

26 Die britische Haltung kommt in einer Bemerkung des Unterstaatssekretärs im India Office, Sir Arthur Godley, zum Ausdruck: "We don't want Koweit, but we don't want any one else to have it." Zitiert nach Busch, Status of Kuwayt, S. 196.

27 Hierzu Gehrke/Kuhn. Irak, S. $130 \mathrm{ff}$.

28 Das Osmanische Reich beharte energisch auf der Position, daß die Territorialhoheit über Kuwait in Konstantinopel läge und über seine Geschicke letzlich hier entschieden würde. Dessen ungeachtet übte Mubarak faktische Souveränität aus, erhob z.B. Zölle und schloß weitere Verträge mit Großbritannien; vgl. Gehrke/Kuhn. Irak, S. 53 ff., Dokumentenanhang, Dok. 8 (Postvertrag) u. 9 (Pachtvertrag), S. 14 ff.

29 Vgl. ebda., S. 121 ff. Die gleiche Interpretation bei Albaharna, Gulf States, S. 253 f.

30 Vgl. Gehrke/Kuhn. Irak, S. 143 für andere Beispiele aus dem 19. Jh. (z.B. Ägypten, Rumänien). Vgl. auch Josef Matuz: Das Osmanische Reich. Grundlinien seiner Geschichte, Darmstadt 1985, S. 97. 
Kuwait nominell als "autonomer Qaza" bezeichnet. ${ }^{31}$ Dem Scheich wurde vom Osmanischen Reich ausdrücklich völlige administrative Autonomie zugesichert; "die Türkei mußte sich jeder Einflußnahme auf die Angelegenheiten von Kuwait enthalten, einschließlich der Frage der Nachfolge, und sie durfte keine Verwaltungsakte und keine militärischen Maßnahmen in Kuwait vomehmen."32 Mit dieser Konvention erkannte das Osmanische Reich schließlich den seit jeher offensichtlichen Sachverhalt an, daß Kuwait kein gewöhnlicher Verwaltungsbezirk des Reiches war, der Scheich kein gewöhnlicher Beamter, der Maßnahmen der Regierung von Konstantinopel oder gar des Provinzgouverneurs von Basra umzusetzen hatte. Es bestanden nicht die geringsten Hoheitsbefugnisse des Osmanischen Reiches in Kuwait.33

Das Territorium Kuwaits wird in dieser Konvention zum ersten Mal definiert, und zwar "1. als ein halbkreisförmiges Gebiet u m die Stadt Kuwait einschließlich der Inseln Warba und Bubijan ..., 2. als eine um dieses Gebiet herumliegende Zone, in der der Scheich die Verwaltungsrechte als türkischer Qaimmaqam wahmehmen sollte."34

31 Die Konvention ist als Dokument Nr. 11 im französischen Original abgedruckt bei Gehrke/Kuhn. Dokumentenanhang, S. 24 ff. Die Autoren interpretieren den Status "autonomer Qaza" zu Recht als eine contradictio in adiecto, die einen Kompromiß zwischen dem türkischen Festhalten an voller Souveränität über Kuwait einerseits und dem faktischen Status der Suzeränität andererseits darstellt, den Großbritannien und vor allem Kuwait allein bereit waren anzuerkennen. "... die englischen Unterhändler (gingen) wohl mit Recht davon aus, daß es nicht darauf ankam, mit welchem Namen man das neue Rechtsverhältnis versah, sondem darauf, welche konkreten Rechte Mubarak eingeräumt wurden." dies., Irak, S. 145.

32 Ebda., S. 145. Der in dieser Konvention festgelegte Status von Kuwait läßt sich daher auch nicht vergleichen mit dem Status des autonomen Sanjaks von Mt. Liban oder dem von Dair az-Zur, die beide ebenfalls aus der normalen Verwaltungshierarchie des Osmanischen Reiches herausgelöst waren, über die der Sultan bzw. das Innenministerium aber dennoch Hoheitsgewalt besaßen. Vgl. Max L. Gross: Ottoman Rule in the Province of Damascus, 1860 - 1909, Diss. (unveröff.), Georgetown University, Wash., D.C., 1979, S. 1 ff. u. S. 51 ff.

33 Ronart/Ronart beschreiben das Verhältnis des Osmanischen Reiches zu Kuwait in dieser Phase generalisierend, aber treffend: "... (die) Manifestation der Souveränität des Sultans (blieb) nur noch eine von der osmanischen Regierung aus Prestigegründen aufrechterhaltene Fiktion.", in: Stephan und Nandy Ronart: Lexikon der Arabischen Welt. Ein historisch-politisches Nachschlagewerk, Zürich/München 1972, S. 621.

34 Gehrke/Kuhn. Irak, S. 82. Vgl. dies.: Dokumentenanhang, Dok. 11, S. 24 ff., bes. Artikel 5-7. Trotz der zitierten Autonomie des Scheichs läßt sich gerade durch den Wortlaut dieser Passage eine implizit vorgenommene Anerkennung der Souveränität des Sultans von Konstantinopel in die Konvention hineininterpretieren, wie z.B. bei Pillai/Kumar, Status of Kuwait, S. $120 \mathrm{ff}$., die zur Stützung dieser Interpretation auch andere Punkte der Konvention heranziehen. Zur Auseinandersetzung mit diesem Standpunkt vgl. Gehrke/Kuhn. Irak, S. 144 ff. Scheich Mubarak hatte u.a. aus diesem Grunde große Schwierigkeiten mit der Anerkennung der Konvention. Vgl. ebda., S. 83 f. u. Fn. 31 . 
Der 1. Weltkrieg, an dem das Osmanische Reich auf seiten des Deutschen Reiches und Österreich-Ungams teilnahm, brachte für den Status Kuwaits eine emeute Veränderung. Kurz vor Kriegsausbruch, als türkische Truppenbewegungen im Zweistromland eine Besetzung Kuwaits anzukündigen schienen, erklärte Großbritannien am 3.11.1914 das Scheichtum zu einem "Independent Government under British Protection". Damit war Kuwait faktisch aus dem osmanischen Reichsverband ausgeschieden, auch wenn dies erst nach dem Kriege in rechtliche Formen gegossen wurde. Diese Entwicklung war ganz in Scheich Mubaraks Sinn.35 Konsequenterweise leistete das Scheichtum dem Osmanischen Reich im 1. Weltkrieg auch keine Heeresfolge, wie es einem Vasallen eigentlich obliegt, sondern nahm als Verbündeter Großbritanniens am Krieg gegen das Reich teil.36

Die zweite Phase der Geschichte Kuwaits von 1899 bis zum 1. Weltkrieg ändert, zusammenfassend beurteilt, nichts an dessen Autonomie. Kuwait verblieb unter der Hoheitsgewalt der Al Sabah Dynastie als Vasallenstaat im osmanischen Reichsverband. Als solcher stand er unter britischem Schutz, welcher sich insbesondere auf die Sicherung der inneren Autonomie Kuwaits bezog. Angesichts der unstrittigen Vasallität wurde jedoch erst im Zuge der britischen Erklärung von November 1914 ein halbwegs formalisierter Protektoratsstatus erklärt. Da Kuwaits Unabhängigkeit explizit festgehalten blieb, wurde das Scheichtum jedoch nicht zu einem echten Protektorat; dies gilt unabhängig von der Argumentation, daß diese Unterscheidung unter politischen Gesichtspunkten zumindest für die Zeit bis zum Ende des 2. Weltkrieges durchaus als künstlich gewertet werden kann.37 Diesen Status behielt der Golfstaat bis zum Jahre 1961, als der britische Schutz formal für beendet erklärt wurde.

Saddam Husseins Argumentation, Kuwait sei zur Zeit des Osmanischen Reiches Bestandteil der Provinz Basra gewesen, muß also zurückgewiesen werden. Weder aus dem nominellen Anspruch des Osmanischen Reiches auf volle Souveränität über Kuwait, noch aus der tatsächlichen Vasallität bis 1914 läßt sich die Integration Kuwaits in das Reich von Konstantinopel konstruieren. Unbestritten ist, daß das Britische Empire als imperiale Großmacht in die Geschichte Kuwaits eingegriffen hat; die Frage, ob die Überlebensfähigkeit Kuwaits allein durch Großbritannien gesichert wurde oder ob Kuwait sich der abzeich-

35 Die von Mubarak ungeliebte englisch-osmanische Konvention von 1913 war damit praktisch hinfällig geworden. Für weitere Gründe vgl. Dickson, Kuwait, S. $150 \mathrm{f}$.

$36 \mathrm{Vgl}$. Gehrke/Kuhn. Irak, S. $86 \mathrm{ff}$. (hier auch zur schwankenden Haltung Kuwaits als britischer Verbündeter nach dem Tode Scheich Mubaraks 1915) u. S. 149; Pillai/Kumar, Status of Kuwait, S. 122 ff. für eine eingehendere Diskussion über die Rechtmäßigkeit der britischen Erklärung von 1914.

37 So i.a. die Bewertung des faktischen kuwaitisch-britischen Verhältnisses. Seit 1903 war Großbritannien mit einem "Political Resident" in Kuwait vertreten, der ursprünglich dem Govemment of India unterstand und im wesentlichen die kolonialen Interessen des Empires vertrat. Der zitierte $H$. R. P. Dickson hatte dieses Amt lange Jahre inne. 
nenden Integration in das Osmanische Reich nach 1871 auch aus eigenen Kräften hätte entziehen können, muß offen bleiben. Unerheblich ist in diesem Zusammenhang jedoch, welche politischen Gründe letztlich für Großbritanniens Engagement ausschlaggebend waren. In jedem Fall ist die Argumentation falsch, daß ein autonomer Staat Kuwait nicht existiert habe.

IV.

Dieser Sachverhalt hat entscheidende Konsequenzen für die Behauptung, Kuwait hätte im Zuge der Neugliederung der politischen Landkarte nach dem 1. Weltkrieg in den Irak integriert werden müssen. Das Osmanische Reich gehörte zu den Verlierem des Krieges. Die Niederlage hatte den Verlust jeglichen Anspruches in den arabischen Provinzen zur Folge; dies bestätigten die Türken in den Verträgen von Sèvres (1920) und Lausanne (1923), wodurch u.a. Kuwait formal aus dem osmanischen Reichsverband entlassen wurde.38

Die Etablierung neuer Staaten mit eigenen Verwaltungsstrukturen wie auch deren Grenzziehung sind im wesentlichen von den Siegermächten Frankreich und Großbritannien durchgeführt worden. Sie handelten dabei offiziell im Auftrage des Völkerbundes, der ihnen Mandate über Syrien und Libanon (Frankreich) sowie über Palästina und Mesopotamien (Großbritannien) verliehen hatte.39 Kuwait ist in diesem Prozeß niemals als Einheit mit dem Mandatsgebiet Mesopotamien behandelt worden, in dem später der Staat Irak entstand, weder im Abkommen von San Remo 1920, noch in den folgenden Verträgen, die die Staatswerdung des Irak und die Festlegung seiner Grenzen im einzelnen formalisierten.40 Dies lag in der Natur der Sache: Kuwait existierte als Staat seit 1899, war seit 1914 unter britischem Schutz unabhängig und hatte am 1. Weltkrieg auf seiten der Briten gegen das Osmanische Reich teilgenommen. Es gehörte folglich nicht zu den durch die Kriegshandlungen besetzten Gebieten Mesopotamiens. Der Zusammenbruch des Osmanischen Reiches führte letztlich zu einem unabhängigen Staat Kuwait, während er Mesopotamien unter britische Verwaltung brachte. Eine Einverleibung Kuwaits in das britische Mandat

$38 \mathrm{Zu}$ den Nachkriegsregelungen und den zitierten Verträgen vgl. George Lenczowski: The Middle East in World Affairs, 4. Aufl., Ithaca/London, 1980, S. 92 ff., und Erich Topf: Die Staatenbildungen in den arabischen Teilen der Türkei seit dem Weltkriege nach Entstehung, Bedeutung und Lebensfähigkeit, Hamburg 1929, hier bes. S. $105 \mathrm{ff}$.

39 Die Übertragung der Mandate gemäß Art. 22 der Satzung des Völkerbundes fand auf der Konferenz von San Remo im April 1920 statt, die verbindlichen vertraglichen Regelungen wurden später (1922) festgelegt. Vgl. Lenczowski, Middle East, S. 93 f.; Topf, Staatenbildungen, Anhang 1, 2, 7 für die Übersetzung der Mandatstexte bzw. des englisch-irakischen Vertrages vom 10.10.1922.

40 Vgl. Lenczowski, Middle East, S. 661 f.; Gehrke/Kuhn. Irak, S. 152 ff.; Albaharna, Gulf States, S. $254 \mathrm{f}$., u. Pillai/Kumar, Status of Kuwait, S. $121 \mathrm{f}$. Übereinstimmend auch die Interpretation des immer noch autoritativen George Antonius: The Arab Awakening. The Story of the Arab National Movement, Beirut 1969 (Reprint), S. 279. 
hätte angesichts der mehrfach bestätigten Unabhängigkeit des Scheichtums sogar einen Rechtsbruch dargestellt. Eine "Wiedervereinigung" unter dem Mandatssystem konnte daher nicht erfolgen; Kuwait und Mesopotamien hatte es als Einheit auch vor dem Kriege nicht gegeben: "... weder England als Protektionsmacht, noch die Türkei, ... noch der Völkerbund waren berechtigt, von sich aus über den Status von Kuwait, der ein eigener Staat war, ohne dessen Zustimmung zu verfügen."41

Die weitere politische Entwicklung verfestigte denProzeß der Konsolidierung zweier unterschiedlicher Staaten Irak und Kuwait. Aufgrund erheblicher Unruhen entschlossen sich die Briten, eine arabische Herrschaft im Mandatsgebiet von Mesopotamien zu errichten. Am 11. Juli 1921 wurde der Staat Irak als Monarchie unter der mit Großbritannien verbündeten Familie der Haschemiten konstituiert. 42 Er umfaßte territorial im wesentlichen das Gebiet der ehemaligen osmanischen Provinzen von Mossul43, Baghdad und Basra, zu denen große Teile der syrisch-irakischen Wüste im Westen traten.

Vor diesem Hintergrund wird ersichtlich, daß der neue Staat Irak keine völkerrechtlichen Ansprüche auf Kuwait etwa nach dem Prinzip der Staatensukzession erheben kann. Durch die Verträge von Sèvres und Lausanne hatte die Türkei auf alle Rechte in ihren ehemaligen Provinzen und Reichsteilen verzichtet, in welcher Form diese auch immer bestanden hatten.44 Irak, als Staat in Teilen dieser Gebiete neu entstanden, kann daher kein Territorium beanspruchen, auf das das Osmanische Reich bereits verzichtet hat. Es gibt somit keine aufrechterhaltenen Ansprüche des Osmanischen Reiches, die im Sinne der Staatensukzession auf Irak hätten übergegangen sein können. 45

Wenn also an der staatlichen Existenz Kuwaits - das als Quasi-Protektorat jedoch unter britischem Schutz verblieb - nicht zu zweif eln war und diese durch die politische Entwicklung auch bestätigt wurde, so blieb dennoch die Frage nach den Grenzen des Staates offen. Die Konvention von 1913, das einzige Dokument mit einem Bezug zur territorialen Frage, war wegen der Kriegsumstände nicht ratifiziert worden, aber auch durch die neue Situation nach dem Kriege insgesamt hinfällig geworden. Das schwierige Unterfangen einer Grenzziehung in der Wüste mußte jedoch nun vor allem in Angriff genommen werden, weil die Ubergriffe wahhabitischer Kämpfer aus dem Innern Arabiens in den Südirak und in kuwai-

41 Gehrke/Kuhn. Irak, S. 157.

42 Hierzu ausführlich Longrigg: Iraq, 1900 - 1950. A Political, Social and Economic History, London/New York/Toronto 1953, S. 99 ff. (Kap. 4 u. 5); Malcom E. Yapp: The Making of the Modem Near East, 1792 - 1923, London/New York 1987, S. 331 ff.

$43 \mathrm{Zu}$ den Problemen um Mossul vgl. Lenczowski, Middle East, S. 69, 93 f.

$44 \mathrm{Vgl}$. Albaharna, Gulf States, S. 255; Pillai/Kumar, Status of Kuwait, S. $127 \mathrm{ff}$.. Hier auch jeweils zur Bedeutung des nicht ratifizierten Vertrages von Sèvres.

45 So auch Pillai/Kumar, Status of Kuwait, S. 128: "Iraq can hardly claim what Turkey had already surrendered." 
tisch beanspruchtes Territorium in den Jahren nach dem Weltkrieg kontinuierlich anhielten. $46 \mathrm{Im}$ Dezember 1922 wurden auf der Konferenz von Uqair am Persischen Golf schließlich die Grenzen zwischen dem Irak und Najd (dem späteren Saudi-Arabien) und Kuwait und Najd festgelegt. Gleichzeitig wurden zwei neutrale Zonen geschaffen, von denen eine - die irakisch-saudische - noch heute besteht. 47

Während Abd al-Aziz ibn Saud die Interessen des Najd selbständig wahmahm, wurden der Irak und Kuwait von britischen Verwaltungsbeamten vertreten. Kuwait als die am schwächsten vertretene Partei verlor nach den Beschlüssen der Konferenz an Ibn Saud ca. 2/3 des Territoriums, das in der Konvention von 1913 Scheich Mubarak als Verwaltungsbezirk zuerkannt worden war.48 Erst einige Monate später, im April 1923, wurde durch den britischen Hochkommissar im Irak, Sir Percy Cox, und Scheich Ahmed von Kuwait Einvernehmen über die irakisch-kuwaitische Grenze erzielt. Sie sollte im wesentlichen dem Verlauf entsprechen, der 1913 als Grenze zwischen Kuwait und der damaligen osmanischen Provinz von Basra definiert worden war, einschließlich der Bestimmungen, daß die Inseln Warba und Bubiyan zu Kuwait gehörten, Umm Qasr und die Halbinsel Fao hingegen nicht.49 Diese Grenzfestlegungen von 1922/23 hatten bis August 1990 Bestand50, auch wenn in weiten Abschnitten eine genaue Demarkation nie erfolgte.

Von irakischer Seite ist in den folgenden Jahrzehnten immer wieder die Tatsache moniert worden, daß die Grenzen des Irak von der britischen Mandatsmacht festgelegt wurden, der Irak selbst an diesem Prozeß also nicht beteiligt war. Abgesehen davon, daß dieser Vorgang unter den damaligen gültigen völkerrechtlichen Bedingungen des Mandatssystems durchaus legal war 51 , wird dadurch die grundsätzliche Legitimität der staatlichen Existenz Kuwaits nicht in Frage gestellt; die jeweilige Staatswerdung Kuwaits und Mesopotamients/Iraks war, wie dargestellt, seit spätestens 1899 getrennt verlaufen.

Im Sommer 1932 hat der irakische Premierminister Nuri as-Said schließlich im Zuge der Verhandlungen, die zum Abzug der Briten und zur vollständigen Unabhängigkeit Iraks führten, in einem Notenwechsel mit dem Scheich von Kuwait die ein Jahrzehnt zuvor

46 Vgl. Topf, Staatenbildungen, S. $114 \mathrm{ff}$;; Dickson, Kuwait, S. $243 \mathrm{ff}$.

47 Zur Konferenz von Uqair die Darstellung Dicksons, Kuwait, S. 270 ff., der hieran teilnahn, und Gehrke/Kuhn. Irak, S. 90 ff.; Text des Vertrages bei Gehrke/Kuhn. Dokumentenanhang, Dok. 4, S. $8 \mathrm{f}$., und bei Topf, Anhang 16, S. $232 \mathrm{f}$. Vgl. auch Dep. of State, Bureau of Intelligence and Research (Ed.): International Boundary Study, no. 103, Sep. 15, 1970: Kuwait - Saudi-Arabia Boundary (mit detaillierten Informationen zur Teilung der zweiten neutralen Zone 1965/69) u. Int. Boundary Study, no. 111, June 1, 1971: Iraq - Saudi-Arabia Boundary.

$48 \mathrm{Zu}$ den Reaktionen in Kuwait auf diese Beschlüsse vgl. Dickson, Kuwait, S. $278 \mathrm{ff}$.

49 Vgl. ebda., und Gehrke/Kuhn. Irak, S. 92 f.

50 Mit Ausnahme der neutralen Zone zwischen Kuwait und Najd/Saudi-Arabien; vgl. Fn. 47.

$51 \mathrm{Vgl}$. Gehrke/Kuhn. Irak, S. 159 ff. 
beschlossene Grenzziehung bestätigt. Dieser Schritt setzte in gewisser Weise einen formalen Schlußpunkt unter die seit 1899 getrennt verlaufenen Staatsbildungsprozesse Kuwaits und Iraks. Auch der Irak hatte 1932 somit Grenzen und Souveränität Kuwaits völkerrechtlich anerkannt. 52

\section{V.}

In den folgenden Jahrzehnten ist diese Anerkennung wiederholt in Zweifel gezogen worden. Dafür waren allerdings weniger historisch-rechtliche Gründe ausschlaggebend, die das Verhältnis Kuwait - Irak in den Mittelpunkt stellten. Vielmehr wurde zum einen das Aufgehen Kuwaits in den Irak oder einen noch größeren arabischen Staat auf der Grundlage der unter den arabischen Massen attraktiven ideologischen Strömungen des arabischen Nationalismus und der späteren sozialreformerischen Spielarten des Panarabismus gefordert.53 Thematisiert wurde also nicht speziell die Legitimität Kuwaits, sondem die des gesamten arabischen Staatensystems, das nach dem 1. Weltkrieg durch die Mandatsmächte geschaffen und in der arabischen Welt als künstlich und illegitim perzipiert wurde. In Kuwait selbst gab es kontinuierlich politische Kräfte, die den Anschluß an Irak forderten.54 Ein zweiter Aspekt ist politisch-geographischer Natur. Da die Küstenlinie des Irak für den Bau größerer Tiefwasserhäfen nicht geeignet ist, muß der Irak erhebliche Nachteile beim Olexport in Kauf nehmen.55 Er ist auf Pipelines angewiesen, die zu Häfen anderer Staaten führen, was eine strategische Verwundbarkeit der irakischen Wirtschaft und der gesamten Regionalpolitik bedeutet. 56 Ein dritter, rein politischer Grund ist der Olreichtum Kuwaits, der nach dem 2. Weltkrieg in seinem gesamten Umfang sichtbar wurde und irakische Begehrlichkeiten weckte. Es bleibt allerdings festzuhalten, daß der Irak während der Phase

52 Texte des Notenwechsels bei Albaharna, Gulf States, Appendix 14, S. 383.

53 Vgl. z.B. Alber Hourani: Arabic Thought in the Liberal Age, 1798 - 1939, Cambridge et al. 1988 (Reprint), S. $260 \mathrm{ff}$.

54 So z.B. bereits in den dreißiger Jahren, als ein kurzzeitig bestehender Legislativer Rat für den Anschluß an Irak plädierte; vgl. Gehrke/Kunh, Irak, S. 95 ff. Bis in die jüngste Vergangenheit vertraten bestimmte Oppositionsgruppen in Kuwait panarabisches Gedankengut, das in einem Anschluß an Irak den ersten Schritt zur gesamtarabischen Einheit sah. Zugleich bildeten derartige Forderungen immer auch ein Instrument in der innenpolitischen Auseinandersetzung mit der Herrscherdynastie der Al Sabah.

55 Vgl. Alasdair Drysdale / Gerald Blake: The Middle East and North Africa. A Political Geography, New York/Oxford 1985, S. 112 f.

56 So ist die Pipeline durch Syrien, um die es schon in den siebziger Jahren Konflikte gegeben hatte, seit April 1982 geschlossen; vgl. Eberhard Kienle: Ba'th vs. Ba'th. The Conflict between Syria and Iraq 1968 - 1989, London/New York 1990, S. 65 f., S. 112 f., S. 165. 
der haschemitischen Dynastie (bis 1958) keine völkerrechtlichen Ansprüche auf Kuwait als Ganzes geltend gemacht hat. 57

Am 19.6.1961 erlangte Kuwait durch die einvernehmliche Kündigung des Vertrages mit Großbritannien von 1899 die in jedem Sinne vollständige Unabhängigkeit.58 Nur wenige Tage danach erhob der damalige irakische Staatschef, Oberst Abd al-Karim Qassem, die Forderung nach der Integration Kuwaits in den Irak.59 Wie 29 Jahre später Saddam Hussein versuchte auch Qassem, seine Ansprüche mit dem Hinweis auf Kuwaits angebliche frühere Zugehörigkeit zur osmanischen Provinz von Basra zu legitimieren; die Rechtmäßigkeit der autonomen und souveränen Entwicklung eines kuwaitischen Staates seit 1899 erkannte er nicht an. Die Befürchtungen, Qassem könnte versuchen, seinen Anspruch militärisch durchzusetzen, führten im Sommer und Herbst 1961 zu einer internationalen Krise, in deren Verlauf britische Truppen und solche anderer arabischer Staaten zur Sicherung der kuwaitischen Unabhängigkeit in den Golfstaat entsandt wurden. Militärische Maßnahmen unterblieben daraufhin zwar, aber der Irak konnte - mit Hilfe der UdSSR - die Aufnahme Kuwaits in die UNO für ca. zwei Jahre verzögem. 60

Unter veränderten politischen Rahmenbedingungen trat im Sommer 1963, ein halbes Jahr nach einem emeuten Machtwechsel im Irak (8.2.1963)61, das bilaterale Verhältnis in eine neue Phase. Nachdem die UdSSR ihren Widerstand gegen eine Aufnahme Kuwaits in die UNO aufgegeben hatte62 - Kuwait wurde am 7.5.1963 als 111. Staat in die Weltstaatengemeinschaft aufgenommen -, sah die neue irakische Regierung keine erfolgversprechende Möglichkeit mehr, die Souveränität Kuwaits weiterhin in Zweifel zu ziehen. Kontakte auf Regierungsebene zwischen beiden Staaten wurden aufgenommen. Nachdem das kuwaitische Parlament am 25.9.1963 ein Darlehen in Höhe von 30 Mio. Dinar an Irak bewilligt hatte, wurde am 4.10.1963 in Baghdad ein Abkommen unterzeichnet, "... demzufolge der

57 Auch die Pläne des irakischen Premierministers Nuri as-Said zur Errichtung einer Konföderation zwischen Irak und Jordanien mit Kuwait als Partner 1958 thematisieren nicht die Legitimität der Existenz Kuwaits, sondem behandeln die grundsätzliche Frage nach der Struktur des arabischen Staatensystems.

58 Vgl. den diesbezüglichen Notenwechsel zwischen Großbritannien und Kuwait vom 19.6.1961, abgedruckt bei: Albaharna, Gulf States, Appendix 11, S. $374 \mathrm{f}$. Von britischer Seite wurde das andauemde Vertragsverhältnis von 1899 als "inconsistent with the sovereignty and independence of Kuwait" bezeichnet, zitient nach Pillai/Kumar, Status of Kuwait, S. 114.

59 Vgl. z.B. Majid Khadduri: Republican Iraq, London 1969, S. 233 ff.; Uriel Dann: Iraq under Qassem. A Political History 1958 - 1963, New York 1969, S. 352 f.

60 In die Arabische Liga wurde Kuwait demgegenüber bereits im Juli 1961, einen Monat nach Beendigung des Vertrages von 1899 mit Großbritannien, aufgenommen. $\mathrm{Zu}$ den Abläufen vgl. Lenczowski, Middle East, S. 662, 667.

61 Vgl. Phebe Marr: The modem History of Iraq, Boulder/London 1985, S. $153 \mathrm{ff}$.

62 Hintergrund war die Abkühlung des sowjetisch-irakischen Verhältnisses aufgrund der massiven Verfolgung irakischer Kommunisten durch das neue, baathistisch dominierte Regime. 
Irak die Unabhängigkeit und vollständige Souverănität des Staates Kuwait in seinen gegenwärtigen Grenzen anerkannte und sich beide Staaten zur brüderlichen Zusammenarbeit und Errichtung der gesamtarabischen Einheit verpflichteten ..."63 Dieser Vertrag wurde auf irakischer Seite im übrigen von Premierminister Hassan al-Bakr unterzeichnet; al-Bakr gehörte der Baath-Partei an, war Förderer Saddam Husseins und nach einem weiteren Umsturz von 1968 bis 1979 auch dessen direkter Vorgänger als irakischer Präsident.

Dennoch kam die Kuwait-Frage auch in der Folgezeit mehrfach wieder auf die politische Tagesordnung. Im März 1973 kam es sogar zu gewaltsamen Aktionen, in deren Verlauf irakisches Militär kuwaitische Grenzposten angriff und für einige Wochen kuwaitisches Territorium besetzte.64 Erst massiver Protest innerhalb der Arabischen Liga und die Aufstellung von ca. 20.000 saudischen Soldaten in Kuwait veranlaßte den Irak, die eigenen Truppen wieder zurückzuziehen. Die irakischen Forderungen während dieser Krise bezogen sich auf den Verlauf der Grenze, nicht auf die grundsätzliche Legitimität der staatlichen Existenz Kuwaits. So wurden u.a. die beiden Inseln Warba und Bubiyan beansprucht, die nach irakischer Darstellung für den Zugang und Ausbau des Hafens Umm Qasr notwendig waren. Die Vorgänge von 1973 führten zu einer nachhaltigen Verschlechterung der Beziehungen beider Staaten; die Grenzen zwischen ihnen blieben bis 1977 geschlossen.65

Nachdem Ende der siebziger Jahre eine langfrisige Regelung möglich erschienen war, veränderte der im September 1980 ausgebrochene Krieg zwischen Irak und Iran die Situation emeut. Als bereits in der ersten Phase der militärischen Auseinandersetzungen der wichtigste Zugang Iraks zum Golf, der Flußlauf des Shatt al-Arab, unpassierbar wurde, wurde die strategische Bedeutung der kuwaitischen Inseln Warba und Bubiyan akut. Saddam Hussein, mittlerweile Präsident des Irak und verantwortlich für den Ausbruch des Krieges, emeuerte daher den Anspruch auf diese Inseln. Weitergehende Forderungen auf ganz Kuwait wurden jedoch nicht erhoben. Die Gewährung von Krediten in Milliardenhöhe zur Deckung der irakischen Kriegskosten durch Kuwait und andere arabische Golfstaaten verhinderten jedoch, daß diese territorialen Dispute weiter verfolgt wurden. 66

Bereits zwei Jahre nach Beendigung des ersten Golfkrieges im Sommer 1988 fand dann der Uberfall und die Annexion Kuwaits statt, zu dessen Legitimation die historisch-rechtlichen Argumente wieder belebt wurden. Die politischen Motive Saddam Husseins sind in diesem

63 Gehrke/Kuhn, Irak, S. 118. Der Vertragstext ist abgedruckt bei Albaharna, Gulf States, Appendix 15, S. $384 \mathrm{f}$.

64 Vgl. Archiv der Gegenwart (AdG), 1973, S. 17907 f. Zu den politischen Hintergründen vgl. z.B. Edmund Ghareeb: Iraq in the Gulf, in: FrederickW. Axelgard (Ed.): Iraq in Transition. A Political, Economic and Strategic Perspective, Boulder/London 1986, S. 59-83, hier S. 64 f.

65 Vgl. AdG 1977, S. 21172.

66 Vgl. zum Gesamtzusammenhang: Shahram Chubin / Charles Tripp: Irand and Iraq at War, London 1988, S. 152 ff.; auch Liesl Graz: The Turbulent Gulf, London/New York 1990, S. 86 f. 
Beitrag nicht eingehender darzustellen. Sie liegen, wie eingangs bereits erwähnt, einerseits in der katastrophalen finanziellen Situation des Irak nach achtjährigem Krieg gegen den Iran; mit der Annexion Kuwaits sollte einer der größten Gläubiger des Irak beseitigt werden. Zum anderen sind sie in dem Bestreben Saddam Husseins zu suchen, den Nahen Osten, zumindest aber die Golfregion unter seiner Führung neu zu ordnen.67

VI.

Die Darstellung der historischen und völkerrechtlichen Aspekte der derzeitigen Golfkrise führen zu folgenden Antworten auf die eingangs gestellten Leitfragen:

1. Die Argumentation, Kuwait sei ein integraler Bestandteil des Osmanischen Reiches gewesen, ist nicht haltbar. Kuwait hat als Vasallenstaat zum osmanischen Reichsverband gehört, nicht aber als Verwaltungsbezirk zur Provinz von Basra. Kuwait war in der Endphase des Osmanischen Reiches, als ein Staat Irak noch nicht einmal in seinen Grundzügen existierte, bereits ein Staat unter britischem Schutz. Der Irak kann somit keine Ansprüche geltend machen, die sich auf eine frühere Zugehörigkeit Kuwaits zum Osmanischen Reich stützen.

2. Aus diesem Grunde ist die Argumentation, Kuwait hätte nach dem 1. Weltkrieg in den Irak als Nachfolgestaat des Osmanischen Reiches integriert werden müssen, nicht stichhaltig. In den Verträgen von Sèvres und Lausanne hatte das Osmanische Reich auf alle Rechte in den ehemaligen arabischen Reichsteilen verzichtet. Ansprüche des Irak, der in Teilen dieser Erbmasse als Staat neu entstand, auf den bereits bestehenden Staat Kuwait sind daher nicht aufrecht zu erhalten.

Diese Ansprüche wären auch dann nicht haltbar, wollte man - fälschlicherweise! - eine formale Integration Kuwaits in die osmanische Provinz Basra vor dem Kriege unterstellen. Die territoriale Verwaltungsstruktur des Osmanischen Reiches - die häufigen Veränderungen unterworfen war - hatte für die gebietsmäßige Definition der neu entstandenen arabischen Staaten nach dem 1. Weltkrieg keine entscheidende Bedeutung. 68

3. Die Politik Großbritanniens nach $\mathbf{1 8 9 9}$ hat einen status quo garantiert: die Autonomie Kuwaits. Hingegen ist Kuwait nicht von den Briten gegründet worden. Es ist unbestrit-

67 Vgl. Fn. 2.

68 Dies wird besonders an der territorialen Neugestaltung Syriens und Libanons durch die französische Mandatsmacht deutlich; vgl. Longrigg, Syria and Lebanon under French Mandate, London/ New York/Toronto 1958, z.B. S. 123 ff. 
ten, daß Großbritannien koloniale Eigeninteressen in der Region vertrat. Daraus lassen sich in der hier behandelten Frage historisch-rechtlicher Ansprüche Iraks auf Kuwait jedoch keine Argumente zur Stützung der Position Saddam Husseins ableiten.

4. Der Irak selbst hat Kuwaits Unabhängigkeit zweimal formal anerkannt, im Notenwechsel von 1932 und im Abkommen von 1963. Letzteres ist von einem baathistischen Regime unterzeichnet worden, so daß auch eine mögliche Argumentation entfällt, die Anerkennung sei von Regimen erfolgt, die faktisch unter kolonialer Abhängigkeit gestanden hätten. Zwischen 1921 bzw. 1932 und 1961 und nach 1963 hat der Irak weder vor dem Völkerbund noch vor der UNO dieser Anerkennung zuwiderlaufende Ansprüche kontinuierlich vertreten. Er hat 1964 die Mitgliedschaft Kuwaits in der Arabischen Liga akzeptiert und in den knapp drei Jahrzehnten danach Beziehungen zu seinem Nachbarn gepflegt, die - trotz aller Grenzprobleme - denen souveräner Staaten entsprachen.

5. Die haschemitischen Föderations- und Konföderationspläne in den dreißiger und fünfziger Jahren behandelten nicht zentral das kuwaitisch-irakische Verhältnis. Bei ihnen ging es - unter jeweils unterschiedlichen, regionalen Rahmenbedingungen - um eine Neustrukturierung des nahöstlichen Staatensystems insgesamt. Ein kontinuierlich erhobener Anspruch Iraks auf Kuwait läßt sich aus diesen Plänen nicht konstruieren.

6. Das periodenweise virulente Kuwait-Problem ist von irakischer Seite mit unterschiedlicher Argumentation und unterschiedlichen Zielsetzungen behandelt worden. Diese haben sich einmal auf die Legitimität Kuwaits als Ganzes bezogen (Qassems KuwaitKrise 1961-63), später lediglich auf Einzelaspekte der Grenzziehung (1973, 1980). Die irakische Position war also in der Vergangenheit insgesamt wechselhaft. "Besonders in Krisenzeiten wurden vom Irak ... territoriale Ansprüche erhoben, die entweder der Erpressung Kuwaits dienten oder das Ziel verfolgten, einen Zugang zum Golf auf Kosten Kuwaits zu erobern."69

Zusammengenommen lassen diese Ergebnisse nur den Schluß zu, daß Invasion und Annexion Kuwaits durch den Irak weder nach den Regeln des geltenden Völkerrechts noch vor dem Hintergrund der historischen Entwicklung zu rechtfertigen sind. Die Argumentation Saddam Husseins ist nicht stichhaltig, seine Aktion entbehrt jeglicher Legitimität. 
VII.

Trotz dieses Sachverhalts hat die Aktion Saddam Husseins unter der arabischen Bevölkerung des Nahen Ostens sehr viel Zustimmung und Unterstützung erfahren. Die für viele westliche Beobachter überraschende und schwer nachvollziehbare Parteinahme der sogenannten "arabischen Massen" für die Sache des irakischen Diktators schließt die Akzeptanz seiner Argumentation mit ein. Es bleibt daher abschließend, thesenartig auf einige dafür verantwortliche Gründe zu verweisen.

1. Die Olvorkommen haben Kuwait zu einem der reichsten Länder der Welt gemacht. Sie ermöglichen den Kuwaitis - wie den meisten anderen Bewohnern der arabischen Halbinsel - einen Lebensstandard, der in der übrigen arabischen Welt nicht im entferntesten erreicht wird. Die "arabischen Massen" empfinden den Reichtum Kuwaits jedoch als ungerecht, da ihnen das Öl als Besitz aller Araber gilt; konsequenterweise müsse der damit zu erzielende Gewinn auch allen Arabem zustehen. Demgegenüber scheinen die reichen Olländer jedoch lediglich die Erhöhung des eigenen Wohlstandes zu betreiben. Die finanziellen Unterstützungen für die ärmeren "arabischen Brüder" werden als ungenügend gewertet, mehr noch: als Almosen und Trinkgelder, die lediglich als Vorwand dienen, um vom eigenen Reichtum abzuienken. Daher sind die Kuwaitis und die Bewohner der anderen reichen Olstaaten auf der arabischen Halbinsel unbeliebt, ja teilweise verhaßt. Vor diesem Hintergrund trägt der Schritt Saddam Husseins in der Perzeption vieler Araber somit zur sozialen Gerechtigkeit im Nahen Osten bei, da er zumindest potentiell den bislang benachteiligten Arabern die Reichtümer des Öls erschließt.

2. Darüber hinaus betrachtet die arabische Bevölkerung des Fruchtbaren Halbmondes und Nordafrikas die Bewohner der Halbinsel in der Regel als Beduinen, deren Kulturstufe als der eigenen weit unterlegen bewertet wird. Dies komme beispielsweise in dem hohen Gastarbeiteranteil zum Ausdruck: Die Kuwaitis selbst seien nicht in der Lage, ihren Olreichtum eigenhändig umzusetzen. Für alle Arbeiten - von der Olförderung bis zur Autoreparatur - benötigten sie Hilfe von außen. Diese kulturelle Konfliktlinie trägt ebenfalls zur perzipierten Legitimität der irakischen Aktion bei.

3. Die Hoffnungen der Araber, nach dem Untergang des Osmanischen Reiches am Ende des 1. Weltkrieges einen gesamtarabischen Staat errichten zu können, der an große historische Vorbilder anknüpft, haben sich nicht erfüllt. Die seither bestehende tiefgreifende politische und staatliche Spaltung der Araber, für die nicht-arabische Staaten verantwortlich sind, wird im allgemeinen aus traumatisch empfunden. Gleichzeitig wird die Fiktion aufrecht erhalten, daß die Aufhebung dieser Spaltung durch die Etablierung eines gesamtarabischen Staates, der erste, notwendige Schritt sei, um die diversen Krisen der arabischen Welt wirkungsvoll zu überwinden. Wer, wie Saddam Hussein durch 
die Annexion Kuwaits, einen Schritt in diese Richtung geht, trägt zur Erreichung dieses zentralen gesamtarabischen Kemanliegens bei, unabhängig von seinen eigentlichen politischen Zielen. Vor diesem Hintergrund ist es unerheblich, ob Kuwait als autonomer oder souveräner Staat seit 1899 oder 1961 bestanden hat. Die Existenz Kuwaits wird in jedem Fall als Ergebnis der Kolonialpolitik Großbritanniens gesehen, das wiederum einer der Hauptschuldigen an den Spaltungen in der arabischen Welt sei.

4. An dieser Auffassung ändert auch die Tatsache nichts, daß Kuwaitnach den Regeln des Völkerrechts ein legitimes Existenzrecht hat. Das Völkerrecht wird im Zweifelsfalle als ein von europäischen Kolonialmächten entwickeltes Instrument betrachtet, das nach Belieben von diesen zur Unterdrückung - und das heißt in diesem Fall: zur Beibehaltung der Spaltung - der arabischen Welt eingesetzt wird.

5. In der Perzeption der arabischen Massen hat Saddam Hussein also einen Schritt getan, der sich gegen das Unrecht wendet, das Arabern von Nicht-Arabem angetan wurde. Die Verknüpfung mit dem Palästinenserproblem durch die Forderung nach einem Abzug Israels aus den besetzten Gebieten bestärkt diese Einschätzung. Saddam Hussein hat sich damit zum Sachwalter gesamtarabischer Interessen gemacht; die Ausrufung des Heiligen Krieges gegen die von den USA gefürten Truppen der anti-irakischen Allianz hat dem eine weitere, religiös untermauerte Dimension hinzugefügt. Dies gilt trotz der Tatsache, daß ihm für die Ausrufung des Jihads jegliche islamische Legitimität fehlt.

Die Krise am Golf ist daher nicht allein mit historischen und völkerrechtlichen Argumenten zu erfassen - und schon gar nicht zu lösen. Sie stellt vielmehr einen politischen Konflikt dar, der letztlich seine wahren Hintergründe in der fehlenden Struktur einer im Nahen Osten allgemein akzeptierten regionalen Ordnung hat. 


\section{ABSTRACTS}

The Recent Developments Concerning the Constitution of the Islamic Republic of Iran

By Asghar Schirazi

The article examines the question of whether the revision of the constitution of the Islamic Republic of Iran undertaken between April and June 1989 was the appropriate instrument for solving the contradictions that have been responsible for the failures of the state leadership. By and large the answer is negative, as it merely served to decide who should succeed Chomeini and how his successors were to share power. It was triggered off when Grand Ayatollah Motazeri, Chomeini's designated successor, resigned. As there was no other Grand Ayatollah acceptable to the regime who could replace him, the condition was dropped that the head of state had to be a leader in religious affairs, too. The problems caused by a prime minister and a president both occupying the top of the executive branch were solved in favour of Johhat ol-Eslam Rafsanjani, who was known to want to stand as candidate for the soon-to-be-vacated office of president. The Determination Council, set up by decree in February 1988, was incorporated into the constitution as an additional body limiting the legislative authority of Parliament. The separation of leadership in religion and state was a further step towards freeing the Islamic Republic from its religious destiny. The division of power after the death of Chomeini was not equitable and thus increased tension amongst his successors.

\section{The Historical Background of the Iraqi Territorial Demands on Kuwait}

\section{By René Klaff}

Iraqi President Saddam Hussein's attempt to annex Kuwait and incorporate the small neighbour state into Iraq as its 19th province has not been successful. It caused global resistance, as a consequence of which Iraq was finally driven out of Kuwait by an international alliance whose military capabilities and internal composition was without precedence. In order to fully understand the invasion of August 1990, one has to analyse not only the mere political and strategic reasons that were responsible for the Iraqi President's bold move. Saddam Hussein argued that Kuwait was able to become a sovereign 
state only because of Great Britain's colonial politics, and that Kuwait has always been an integral part of Iraq. Therefore, this article discusses the historical and legal aspects which were brought forward by Saddam Hussein in order to legitimize the invasion and the attempt to incorporate Kuwait into Iraq. Three questions are crucial in this respect:

1. Was Kuwait an integral part of the Ottoman province of Basra?

2. Are there any legitimate claims of Iraq, as a successor state of the Ottoman Empire, to Kuwait?

3. How has Iraq sustained its claims in the past?

The answers to these questions show that Iraqi claims to Kuwait are unfounded.

\section{On the Court System in Libya}

\section{By Hans-Georg Ebert}

As the legal system of Libya is often analysed on the occasion of spectacular or doubtful practices in the judiciary, often with a political background, the institutional structure of the court system itself and its legal basis is seldomly described on a plain legal basis. The article describes the institutional structure in which the courts, attomeys-at-law and court administration work. The article tries to concentrate on the valid statutes and to give an overview, although frequent changes and the dichotomy of state law and sharia make a brief and clear description either impossible or incomplete.

The author also introduces the valid principles governing the legal procedure at court and explains the extent to which the govemment did and does interfere - disrespecting the independence of the judiciary. He concludes that the present Libyan court system is the result of a dual strategy, an attempt to reorganize the legal system integrating the traditional Islamic legal practices and institutions.

\section{Selected Issues Concerning the Operation of Inter-American System for the Protec- tion of Human Rights}

\section{By Antônio Augusto Cançado Trindade}

In the operation of the inter-American system of human rights protection, two issues deserve special attention: the exhaustion of local remedies as a condition of admissibility of 